\title{
Corrigendum
}

\section{Corrigendum to "Mobile Robot Simultaneous Localization and Mapping Based on a Monocular Camera"}

\author{
Songmin Jia, ${ }^{1,2}$ Ke Wang, ${ }^{1,2}$ and Xiuzhi $\mathrm{Li}^{1,2}$ \\ ${ }^{1}$ College of Electronic Information \& Control Engineering, Beijing University of Technology, Beijing 100124, China \\ ${ }^{2}$ Beijing Key Laboratory of Computational Intelligence and Intelligent System, Beijing 100124, China \\ Correspondence should be addressed to Ke Wang; kwangs@sina.com
}

Received 30 November 2016; Accepted 20 December 2016; Published 12 January 2017

Copyright (C) 2017 Songmin Jia et al. This is an open access article distributed under the Creative Commons Attribution License, which permits unrestricted use, distribution, and reproduction in any medium, provided the original work is properly cited.

In the article titled "Mobile Robot Simultaneous Localization and Mapping Based on a Monocular Camera" [1], there were errors in (7), (8), (9), (14), (16), (18), and (19), which should be corrected as follows:

$$
\begin{aligned}
& \arg \min _{\mathbf{x}} \sum\left\|r_{p}(\mathbf{x})\right\|^{2}, \\
& \arg \min _{\mathbf{x}, d} \sum L\left(d(u)-d_{g}(u)\right)\left\|r_{p}(\mathbf{x})\right\|^{2}+\left\|r_{d}(u, d)\right\|^{2}, \\
& L(x)= \begin{cases}1, & |x| \leq \sigma, \\
0, & \text { others, }\end{cases} \\
& \arg \min _{\mathbf{x}} \sum L\left(d-d_{g}\right)\left\|r_{p}(\mathbf{x})\right\|^{2}, \\
& \arg \min _{d} \sum\left\|r_{d}(u, d)\right\|^{2}, \\
& \min _{\mathbf{x}} \sum w\left(r_{p}\right)\left\|r_{p}(\mathbf{x})\right\|^{2} \text {, } \\
& \boldsymbol{\xi}=\underset{\xi}{\arg \min } \sum_{i} w\left(e_{i}\right)\left\|e_{i}\right\|^{2}, \\
& s\left(u_{j}^{i}\right)= \begin{cases}\left\|r_{j}\right\| \leq \sigma_{i}, & \text { accept, } \\
\left\|r_{j}\right\|>\sigma_{i}, & \text { reject, }\end{cases} \\
& r_{j}=\pi\left(H_{i} v_{j}^{i}\right)-u_{j}^{i} .
\end{aligned}
$$

\section{References}

[1] S. Jia, K. Wang, and X. Li, "Mobile robot simultaneous localization and mapping based on a monocular camera," Journal of Robotics, vol. 2016, Article ID 7630340, 11 pages, 2016. 


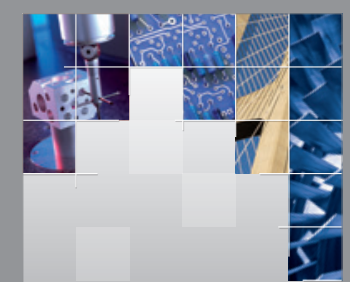

\section{Enfincering}
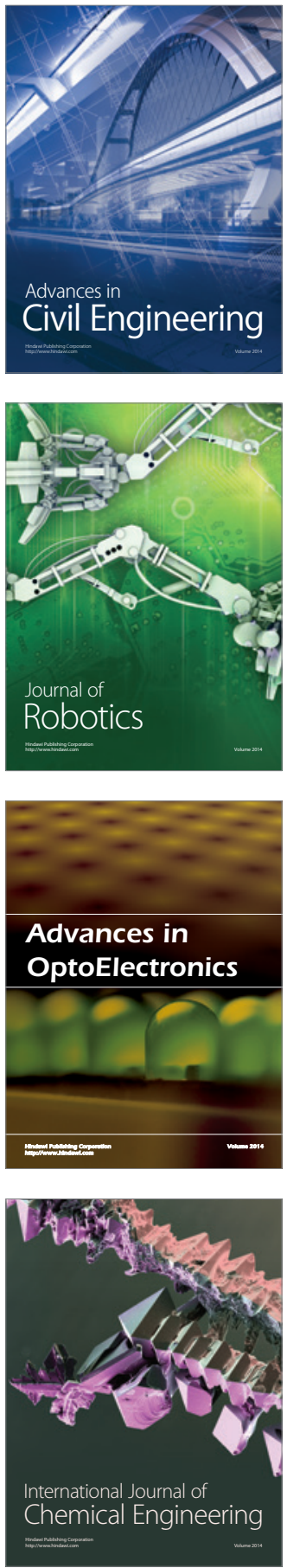

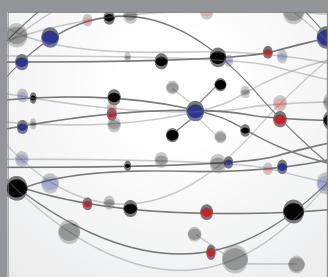

The Scientific World Journal

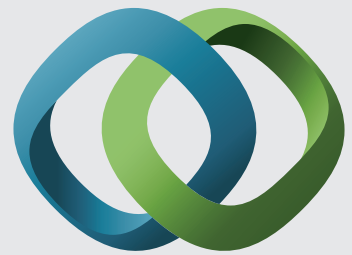

\section{Hindawi}

Submit your manuscripts at

https://www.hindawi.com
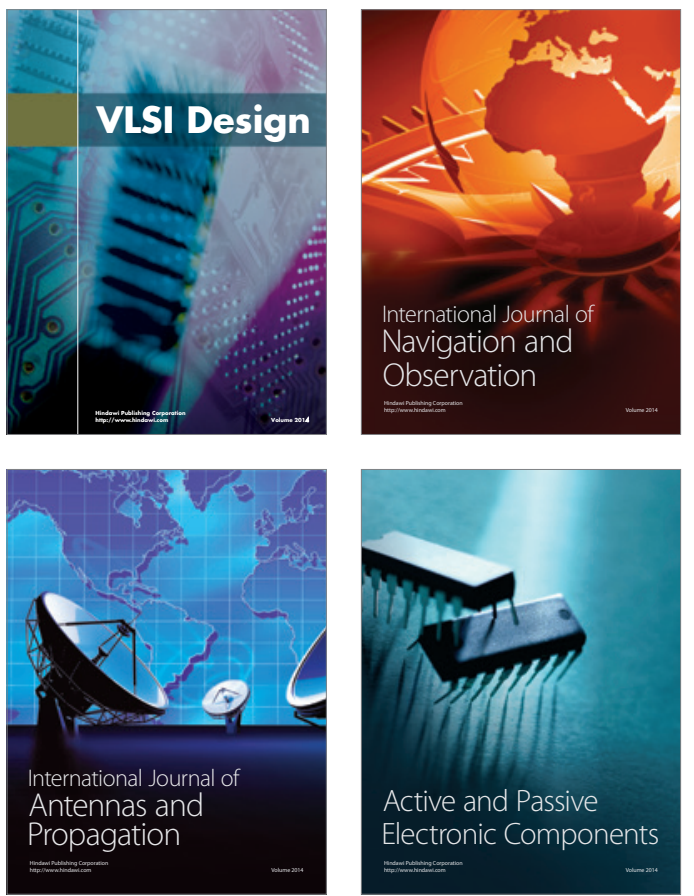
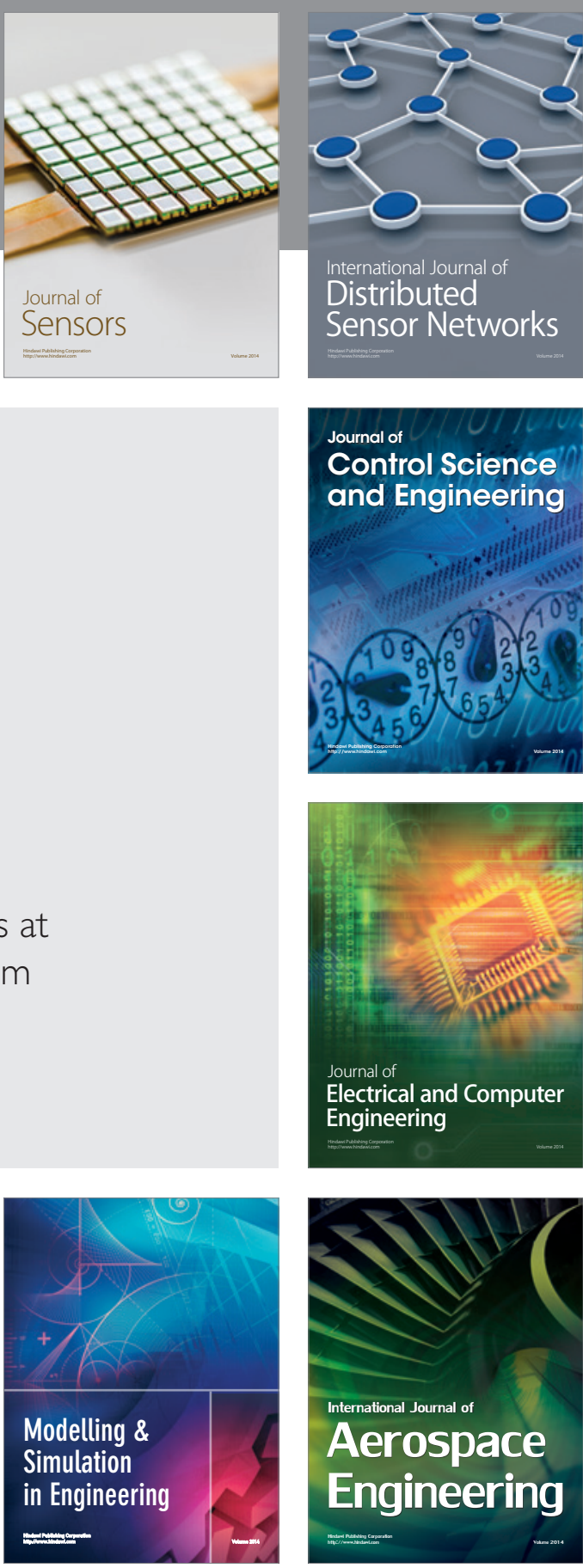

International Journal of

Distributed

Sensor Networks

$-$

Joumal of

Control Science

and Engineering
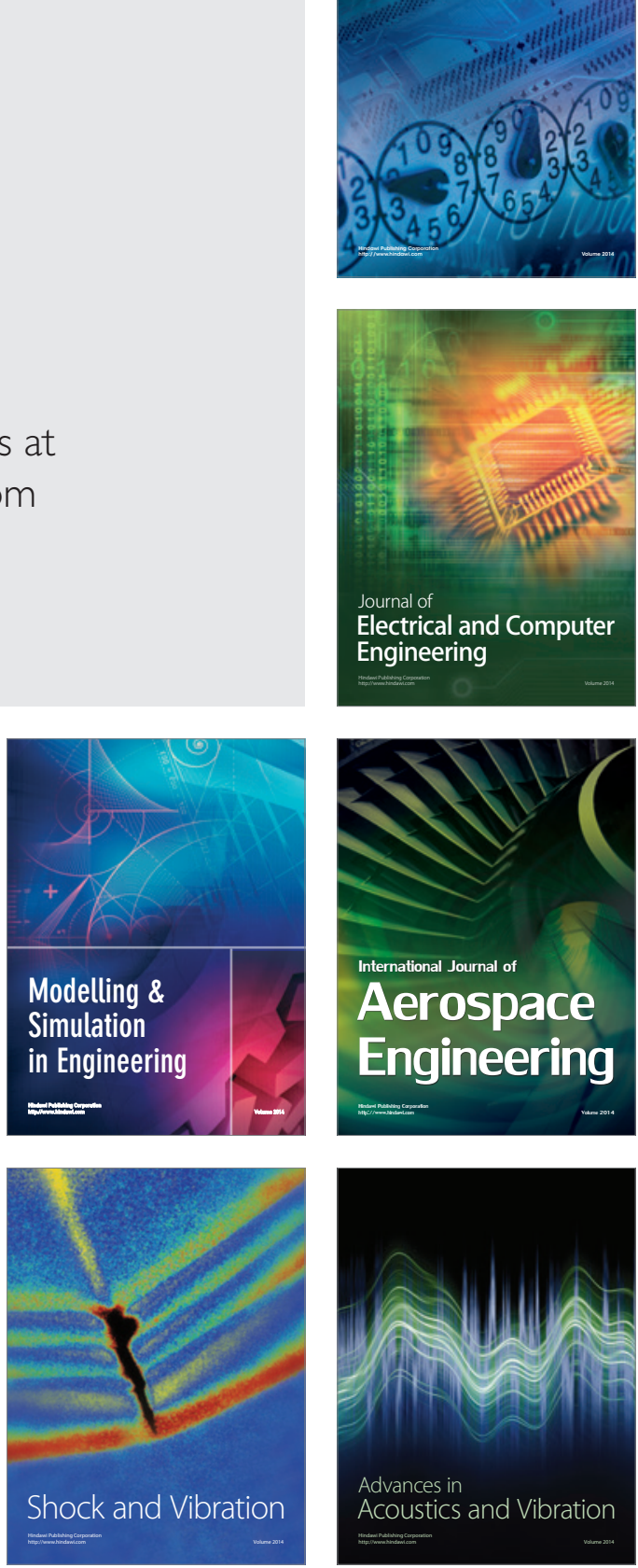